Anuario de Estudios Medievales

43/1, enero-junio de 2013, pp. 323-342

ISSN 0066-5061

doi:10.3989/aem.2013.43.1.11

\title{
DES REGISTRES DE RATIONALITÉ EN CONCURRENCE? EMPIRICA MAGIQUES ET MÉDECINE SCOLASTIQUE
}

\author{
COMPETING LEVELS OF RATIONALITY? \\ MAGICAL EMPIRICA AND SCHOLASTIC MEDICINE
}

\author{
NiCOLAS WEILL-PAROT \\ Université Paris-Est Créteil \\ CRHEC-EA 4392
}

\begin{abstract}
Résumé: On peut interpréter l'opposition récurrente des médecins scolastiques aux vetulae et empirici comme un conflit entre niveaux de rationalité. Sont d'abord distingués trois niveaux de rationalité dans les conceptions de la magie, puis sont étudiées successivement les deux voies de justification rationnelle des empirica intégrés à la médecine savante: celle qui avait recours aux propriétés occultes, celle qui convoquait le pouvoir de l'imagination-dans ce second cas, plutôt le pouvoir de l'imagination sur le corps de celui qui imagine qu'un pouvoir sur un autre corps. Les propriétés occultes semblent offrir une solution plus satisfaisante rationnellement mais aussi peut-être théologiquement. L'article s'achève par l'étude d'un cas: la manière dont le médecin valencien Jeroni Torrella dans l'Opus praeclarum de imaginibus astrologicis (1496-c. 1500) est confronté à un empiricum particulièrement difficile à rationaliser, ce qui illustre, à une date tardive, les enjeux de la rationalisation naturaliste scolastique.
\end{abstract}

Mots-clés: médecine scolastique; rationalité scientifique; empiricum; magie; propriétés occultes; pouvoir de l'imagination.

\begin{abstract}
The recurrent opposition of scholastic physicians to vetulae and empirici can be understood as a conflict between levels of rationality. First, three levels of rationality in the way of thinking magic are set forth; then, two ways of rationally justifying some empirica included in learned medicine are addressed: that which made use of occult properties and that which relied on the power of the imagination -in the latter case, it was rather the power of the imagination on the body of the imagining man than on another body. The occult properties seem to offer a more satisfactory issue, rationally speaking but maybe also theologically. The paper ends with a case study: the way that the valencian physician Jeroni Torrella in the Opus praeclarum de imaginibus astrologicis (1496-c. 1500) faces an empiricum rather uneasy to rationalize -a late testimony of the issue at stake in the scholastic naturalistic rationalization.
\end{abstract}

Keywords: scholastic medicine; scientific rationality; empiricum; magic; occult properties; power of imagination.

\section{SOMMAIRE}

1. Le rejet des empirica magiques par les médecins et les chirurgiens savants.- 2. Ce que peuvent les propriétés occultes et la limite de la destinativité.- 3. La rationalisation des charmes et des incantations par l'imagination et ses limites.- 4. Les hésitations d'un médecin face à un empiricum difficile.- 5. Bibliographie citée. 


\section{LE REJET DES EMPIRICA MAGIQUES PAR LES MÉDECINS ET LES CHIRURGIENS SAVANTS}

Les historiens de la médecine ont depuis longtemps relevé les topoi par lesquels les médecins et les "chirurgiens rationnels" (pour reprendre l'expression de Michael McVaugh ${ }^{1}$ ) s'étaient explicitement et bruyamment démarqués des empirici et autres vetulae, qui employaient des procédés empiriques ouvertement magiques discréditant ainsi la médecine. On connaît la grande sévérité avec laquelle Guy de Chauliac s'en prend à trois auteurs d'ouvrages médicaux remplis de procédés de ce type lorsqu'il signale qu'il n'a pas repris les procédés empiriques ni les incantations trop présents chez Gilbert l'Anglais ou le Thesaurus pauperum de Petrus Hispanus et quand aussi il s'en prend à la Rosa medicinae de John of Gaddesden².

Nicole Oresme, sans être lui-même médecin, fait une critique comparable des tromperies des vetulae qui exercent une concurrence déloyale à l'encontre des médecins: il rapporte qu'un médecin était appelé auprès des malades et déployait tout son art pour les guérir, mais que, dès qu'il était parti, arrivait une femme qui prétendait soigner avec des "duperies" et s'attiraient "les louanges et le mérite de la guérison, alors qu'elle ne faisait rien" et qu'elle détournait à son profit, en somme, le travail du véritable médecin ${ }^{3}$.

La récurrence des critiques savantes de ce type a conduit Lorraine Daston et Katharine Park à interpréter en termes sociologiques cette défiance des savants, "caste sociale et professionnelle", en même temps qu' "épistémologique", à l'encontre du "groupe subordonné" des "vieilles femmes et des artisans" dont ils délégitimaient ainsi le savoir "acquis par d'autres voies (notamment à travers l'expérience)". Elles ont conclu que "l'idéologie sociale de la période identifiait le travail" de ces derniers "avec le travail manuel, qui était fortement stigmatisé dans les cercles de l'élite"4.

${ }^{1}$ M. McVaugh, The Rational Surgery.

2 "Empericas et incantationes parum acceptavi de quibus in Gilbertina et Thesauro pauperum copia invenitur multa" (Guido de Caulhiaco, Chirurgia magna, VII.1.1, p. 391); "Ultimo nunc insurrexit una fatua Rosa anglicana que michi mandata et visa; credidi in ea invenire odorem suavitatis, et inveni fabulas Yspani, Gilberti et Theoderici" (ibidem, cap. singulare, p. 7).

3 "Unde simile audivi et vidi de uno medico qui ad multos egros vocabatur, et postquam recte et bene operatus fuerit, superveniebat una mulier que semper multis egrorum curis ingerebat et faciebat aliquas trufas et sic sibi imponebatur cura et laus que tamen nichil faciebat" (Nicole Oresme, Quodlibet n ${ }^{\circ}$ 7, mss. BnF, lat. 15126, fol. 146, et lat. 15173, fol. 152).

${ }^{4}$ L. Daston, K. Park, Wonders and the Order, pp. 117-118: "All of this was intended to confer on the natural philosopher (together with the theologian and master of theoretical medicine) a virtual monopoly on absolute certainty - something that in the context of medieval education acquired a social and professional as well as an epistemological cast. (...) The intellectual authority of graduates depended in good part on the special claim to certainty that defined the scholastic study of philosophy, which formed the core of the undergraduate curriculum and was 
Sans nier des phénomènes de défiance de groupes socialement et intellectuellement distincts, il n'est pas sûr que la grille et le langage d'une certaine sociologie de la domination -très prisée par les social and cultural studies- soit la mieux placée pour rendre compte de ce qui se joue, au-delà du simple désir d'une profession veillant jalousement sur son monopole scientifique. Il peut être plus pertinent d'interpréter cette confrontation en termes de rationalité, voire de conflits de rationalités -bien que le pluriel ne puisse avoir d'autre sens que celui d'une gradation dans la complexité des processus de rationalisation et dans la diversité des choix des "raisons" effectués par ceux qui pensent.

Les médecins savants se sont efforcés de réaliser un projet scientifique, c'est-à-dire d'appréhender une vérité sur des phénomènes, ceux qui affectent le corps humain, en proposant des explications rationnelles qui formaient entre elles sinon un système, du moins un cadre de référence cohérent; et comme leur discipline articulait un versant théorique et un versant pratique -articulation diversement pensée selon les autorités ${ }^{5}-$, ils ont intégré à leur préoccupation une réflexion sur les moyens pratiques de rétablir la santé du malade. De ce point de vue, les procédés non savants des empirici et des vetulae constituaient à leurs yeux, non seulement, ni prioritairement, une concurrence "sociale", mais une régression rationnelle préjudiciable au travail de métamorphose scientifique qu'ils opéraient sur leur discipline, et qui ne doit pas nécessairement s'expliquer en termes "marxiens", "bourdivins" ou "foucaldiens" de "stratégies de domination" ou autre scie discursive... Les médecins étaient -on peut leur en faire crédit- persuadés du bien-fondé de la science médicale qu'ils avaient apprise, mettaient en œuvre et développaient. Leur défiance à l'égard des procédés plus ou moins magiques des vetulae est comparable à celle que peut avoir aujourd'hui le médecin à l'égard des charlatans ou le biologiste sérieux à l'encontre des créationnistes -il n'y a pas lieu de dénier à ces savants la conviction qu'ils ont eue d'être rationnellement dans le vrai et de façon désintéressée 6 .

Comme l'a montré Danielle Jacquart, la célèbre diatribe de Roger Bacon, dans son De erroribus medicorum, dénonçant la négligence dont faisaient preuve les médecins de son temps à l'égard de l'expérience au profit

considered preparatory of the studies of theology, medicine and law. This view of authority also tended to discount knowledge gained in other ways (notably through experience) by associating it with subordinate groups such as old women and artisans; the social ideology of the period identified their work with manual labor, which was strongly stigmatized in elite circles".

${ }^{5}$ D. Jacquart, La médecine médiévale, p. 415 et suiv.; J. Chandelier, Expérience, expérimentation et connaissance.

${ }^{6}$ Une bonne critique rigoureuse du constructivisme et du relativisme en science a été apportée par P. Boghossian, La peur du savoir. 
de la logique des disputes et des questions, prend sens dans le contexte précis des années 1260-1270 à Paris. Dans les décennies 1240-1270, les maîtres parisiens s'efforçaient d'instituer comme science leur discipline (qui revenait de loin, puisque au haut Moyen Âge, avant les grandes traductions de la fin du XIe siècle et du XIIe siècle, elle était majoritairement une "médecine de recettes") ${ }^{7}$. Autre exemple célèbre, le dominicain Nicolas de Pologne qui, dans les années 1270, s'en prenant aux mêmes tendances théoriques de la médecine savante (sans doute celle de l'université de Montpellier), défendit le recours à un empirisme pur. Il rédigea un recueil d'experimenta dépourvus de toute ébauche même d'explication théorique et explicita ce rejet dans son Antipocras. Il s'agissait d'un retour à un état préscolastique de la médecine et, plus généralement, de la science où les vertus des choses, renvoyées sans plus de précision au pouvoir divin, devaient seulement être constatées ${ }^{8}$.

Est-ce à dire pour autant que les procédés empiriques même magiques étaient dépourvus de raison? Ces pratiques ont bien une rationalité, non celle que la sociologie holiste pense décrypter derrière la conscience des agents sociaux en se référant à un système déterministe de causes, mais celle, par exemple, qu'une autre sociologie, celle de l'individualisme méthodologique de Raymond Boudon, dans la lignée de Weber, reconnaît aux acteurs eux-mêmes dans la conscience qu'ils ont de leur action'. Il s'agit pour cette dernière non de rechercher alors la "rationalité" des causes dépassant et écrasant les agents d'un système social (via, par exemple, le poids de "structures", du reste, mal définies), mais bien de retrouver les "bonnes raisons" qu'ont les acteurs d'un contexte historique donné de croire en l'efficacité de telle ou telle pratique. C'est à cette entreprise théorique que s'est attelé il y a quelques années, Pascal Sanchez, un disciple de Raymond Boudon, en montrant la "rationalité des croyances magiques", c'est-à-dire les "bonnes raisons" que ceux qui pratiquent la magie ont d'adhérer à la croyance dans l'efficacité de leurs opérations ${ }^{10}$. Quelques décennies plus tôt Richard Kieckhefer, lui aussi, avait montré, dans un article consacré à la "rationalité spécifique de la magie" médiévale, que les discours médiévaux de la magie ou sur elle, y compris dans son interprétation démonologique, relevaient bien de l'explication rationnelle ${ }^{11}$.

${ }^{7}$ D. Jacquart, La scolastique médicale, p. 194.

${ }^{8}$ Ibidem, p. 194 note 83; J.W.S. Johnsson, Les "Experimenta Magistri Nicolai"; R. Gansyniec, Brata Mikotaja; W. Eamon, G. Keil, "Plebs amat empirica”; G. Keil, Virtus occulta: Der Begriff.

${ }^{9}$ Voir, par exemple, R. Boudon, La rationalité; idem, Croire et savoir.

${ }^{10} \mathrm{P}$. Sanchez, La rationalité.

${ }^{11}$ R. Kieckhefer, The Specific Rationality. 
Sur ce point, une sociologie non hégémonique, pensant l'action humaine à partir du libre arbitre et du choix rationnel, rejoint l'approche philosophique et historique de la rationalité que je souhaite adopter. J'ai, à plusieurs reprises déjà, proposé une gradation dans la rationalité des pratiques magiques; je me contenterai donc de la rappeler en la précisant pour le sujet qui ici me retient ${ }^{12}$. En premier lieu, il y aurait l'hypothèse liminaire du procédé employé "sans raison": le cas du non-initié utilisant comme pure recette un procédé magique qu'il aurait trouvé par infraction. Ici la seule rationalité, très élémentaire, reposerait simplement sur deux raisonnements. Le premier serait une causalité binaire du type "si je fais cela, alors il se passera ceci". Le second se référerait au cadre de la croyance: "il en sera ainsi, ou il pourra en être ainsi, parce que c'est ce que j'ai entendu ou lu chez une autorité, un magicien". Mais à ce niveau très sommaire de raison, il n'y a aucune explicitation -même à titre d'hypothèse- de la relation par laquelle telle cause (gestes, paroles proférées, substances employées) produit tel effet (le résultat magique). Il me semble que ce cas de figure est celui d'un accomplissement presque parfait de la notion de magie: ce par quoi s'opère (en partie artificiellement) ce qu'on n'explique pas.

Un second niveau de rationalité serait constitué par les théories explicatives proprement magiques, c'est-à-dire celles qui convoquent une causalité ordinaire et habituelle dans le registre de la magie, mais extraordinaire ou du moins surprenante au regard de l'expérience habituelle du monde naturel. Ce peut-être le recours assez flou à un principe de "sympathie" entre les choses rendant compte d'influences à distance. De ce fait, même à un niveau très rudimentaire, des affinités entre l'aspect ou la figure des choses peuvent être pensées ${ }^{13}$. L'autre voie fait intervenir des agents supérieurs intelligents (esprits bénéfiques, maléfiques ou neutres) invités par le rituel mis en œuvre, qui se trouve ainsi conduit au statut de signe, à coopérer à l'opération magique. Dans les deux cas, une causalité, même rudimentaire, est proposée pour combler le chaînon manquant entre la cause exprimée par "je fais ceci" et le résultat formulé par "il se produira ceci". Ce recours à l'explication démonologique est partagé, jusqu'à un certain point, aussi bien par un grand nombre de magiciens que par la critique théologique. La différence entre ces deux points de vue s'expriment ensuite. D'une part, aux arguments apologétiques du magicien (les esprits convoqués sont des anges ou, au contraire, ce sont des démons mais soumis avec l'aide de Dieu) s'oppose la censure implacable

\footnotetext{
${ }^{12}$ Voir notamment N. Weill-Parot, Science et magie.

${ }^{13}$ M. Mauss, H. Hubert, Esquisse d'une théorie, pp. 12-13: sur la sympathie magique et religieuse. Pour une réévaluation de l'importance de ce travail, voir P. Sanchez, La rationalité, pp. 58-76.
} 
du théologien thomiste (les seuls esprits qui peuvent se commettre dans de tels actes sont les démons et les démons ne peuvent être soumis à travers les rituels des magiciens). D'autre part, le magicien semble admettre une modification substantielle des objets magiques mis en jeu dans l'opération, alors que le théologien ne voit là qu'illusion tout entière transmuée en une sémiologie au service du démon ${ }^{14}$.

Enfin, un troisième niveau, supérieur, de la rationalité correspondrait à celle de la science (philosophie naturelle et médecine). Tout en admettant la discrimination théologique à l'encontre d'une magie démoniaque, certains penseurs médiévaux -qu'ils fussent théologiens, médecins ou seulement "philosophes" artiens- furent favorables à l'existence d'une magie naturelle. Cependant ce dernier concept rencontra, dans le cadre philosophique marqué dès le XIIIe siècle par l'aristotélisme universitaire, une série de problèmes liés au paradoxe d'avoir à expliquer, c'est-à-dire à rendre manifestes, les causes d'une opération magique qui, pour rester magique, devait garder une part d'occultation. Pour résoudre ce paradoxe, une minorité céda peut-être aux insatisfaisantes sirènes du pouvoir de l'imagination sur un corps extérieur ${ }^{15}$, mais la plupart s'appuyèrent sur cette remarquable trouvaille qu'étaient les propriétés occultes, lesquelles permettaient de rendre compte d'un phénomène observé sans pour autant révéler le contenu de sa cause, ce qui permettait de maintenir l'opération dans la définition de la magie tout en lui donnant une formulation scientifique. C'est surtout par ce biais que les médecins purent intégrer explicitement -ou plus souvent implicitement- un certain nombre d'empirica à leur pratique thérapeutique.

\section{CE QUE PEUVENT LES PROPRIÉTÉS OCCULTES ET LA LIMITE DE LA DESTINATIVITÉ}

Un point pourrait sembler paradoxal, mais il ne l'est guère: les pratiques magiques qui sont plus facilement intégrables à la rationalité de la médecine savante sont précisément celles qui relèvent originellement du niveau le plus bas de rationalité, les empirica -il était en effet hors de question d'intégrer les procédés suspects de la magie rituelle, pourtant plus savante, à la pratique médicale. Avant même d'entrer dans le détail des justifications, il convient de relever que le choix d'employer tel ou tel empiricum magique pouvait en lui-même procéder d'un raisonnement: j'ai ailleurs cité la récupé-

\footnotetext{
${ }^{14} \mathrm{~N}$. Weill-Parot, Réalité ou vacuité du mal?

${ }^{15}$ Sur cette question, voir notamment: V. Perrone Compagni, "Artificiose operari". L'immaginazione.
} 
ration du sceau astrologique du Serpentaire réputé efficace contre le poison par les auteurs de traités sur la peste, sur la base d'une étiologie assumée par la science médicale: l'empoisonnement de l'air environnant étant considéré comme la cause du fléau ${ }^{16}$.

Michael McVaugh a donné une présentation complète et précise de l'attitude des chirurgiens -mais aussi des médecins- des XIIIe et XIVe siècles vis-à-vis des empirica et des incantationes ${ }^{17}$. On peut résumer à gros traits cette attitude dominante des tenants de la science médicale scolastique parvenue à son accomplissement rationnel, telle qu'on la trouve chez un grand médecin comme Arnaud de Villeneuve ou chez un grand chirurgien comme Guy de Chauliac. D'un côté, ils rejettent les empirica teintés de la magie incantatoire des charmes, de l'autre ils intègrent, en l'assumant pleinement, un grand nombre d'empirica qu'ils encadrent rationnellement.

En effet, tout en dénigrant les médecins versés dans les procédés empiriques comme celui consistant à dire le Pater Noster et à réciter une phrase évoquant l'extraction par Nicodème des clous des mains et des pieds de Jésus-Christ, Guy de Chauliac écrit que, pour ne pas sembler s'écarter du sentier suivi par les autres, il rappellera "le plus brièvement qu' [il] le pourr[a] quelques procédés communs" qu'il utilise de façon courante ${ }^{18}$. Comme le résume M. McVaugh,

apparently it was not simply because of their character as empirica that Guy de Chauliac found these procedures ridiculous, but because prayers or charms played a part in their application ${ }^{19}$.

Il rapproche cette attitude de celle, plus ancienne, d'un Albert le Grand qui, dans le De animalibus, considère que les incantations ne sont pas "raisonnables" 20 . Il cite encore un passage remarquable de la Repetitio super canone vita brevis, où Arnaud de Villeneuve met en avant le critère de la conformité à la raison (utrum sit consonus rationi) pour déterminer si une pratique vulgaire est acceptable ou au contraire superstitieuse $\mathrm{e}^{21}$.

En somme, et l'analyse de McVaugh confirme cette analyse, l'inté-

\footnotetext{
${ }^{16} \mathrm{~N}$. Weill-Parot, La rationalité médicale, pp. 85-86.

${ }^{17}$ M. McVaugh, Incantationes in Late Medieval Surgery. Rappelons aussi: J. Agrimi, Ch. Crisciani, Medici e "vetulae".

18 "Verumptamen ut non videar exire tramitem aliorum, brevius quam potero aliqua magis tamen communia, et michi usitata, remorabo" (Guido de Caulhiaco, Chirurgia magna, VII.1.1, p. 391).

${ }^{19}$ M. McVaugh, Incantationes in Late Medieval Surgery, pp. 328-329.

${ }^{20}$ Ibidem, p. 333.

${ }^{21}$ Ibidem, p. 336.
} 
gration rationnelle des procédés empiriques à la médecine savante pouvait emprunter les deux voies qu'on a mises à jour à propos de la magie naturelle: celle des propriétés occultes et celle de l'imagination -à ceci près (et cela est loin d'être détail) que seul le pouvoir interne de l'imagination sur le propre corps de celui qui imagine est reconnu par la majorité de ces représentants de la pensée médicale dominante. Cela conduit à se demander jusqu'à quelle limite chacun de ces deux types de justification rationnelle des empirica pouvait aller.

La justification par la propriété (occulte) qui soit découle de la forme substantielle d'une espèce, soit survient accidentellement chez un individu particulier au sein d'une espèce donnée ${ }^{22}$, est sans doute le procédé majeur par lequel les empirica comme l'usage d'amulettes, corps naturels naturellement dotés de vertus (dans le premier cas), ou les talismans, objets artificiels recevant un pouvoir magique (dans le second cas), ont été intégrés à la médecine savante. Du reste, aussi bien Arnaud de Villeneuve que Guy de Chauliac furent les promoteurs de l'utilisation d'un talisman purement astrologique, ne tirant son pouvoir que du pouvoir naturel des astres (une "image astrologique"), le sceau du Lion contre les douleurs de rein. Mais cette "image astrologique", qui est l'exemple même de l'empiricum savant accepté quoique véritablement magique, exclut par définition tout ce qui serait "destinatif", c'est-à-dire tout appel via un signe -inscriptions de mots ou de symboles, incantations- à une entité supérieure (ange, démon ou autre esprit) ${ }^{23}$. L'enjeu est double: il est scientifique, puisqu'il s'agit de rester dans le seul cadre des causes naturelles (certes le démon ne fait que les utiliser, mais en tant qu'intelligence il transcende cet ordre); il est aussi théologique, puisque la non-destinativité est, dans le cadre de la pensée scolastique telle qu'elle se développe à partir du XIIIe siècle, le critère essentiel de la licéité. Cette double implication de l'absence de recours au signe dans le procédé empirique constitue, me semble-t-il, la double limite -très étroite- qui tend à exclure l'emploi des incantations et des charmes dans la médecine savante.

Cette étroite solidarité du critère scientifique et du critère théologique n'est pas pour surprendre dans une scolastique qui revendique et assume cette profonde harmonie entre la foi et la raison, bien que la médecine savante se développe assez largement dans un registre autonome et que, si le caractère extra-scientifique ou "non rationnel" d'une incantation est mis en avant dans le discours de ces médecins, la référence à la norme théologique

\footnotetext{
${ }^{22}$ Sur cette distinction: N. Weill-Parot, Astrology, Astral Influences. Parmi les études sur les propriétés occultes, on peut rappeler, entre autres, B.P. Copenhaver, Scholastic Philosophy and Renaissance Magic; K. Hutchison, Dormitive Virtues, Scholastic Qualities; G. Federici Vescovini, La concezione della virtus occulta; S. Giralt, Proprietats: las propiedades ocultas.

${ }^{23}$ N. Weill-Parot, Les "images astrologiques".
} 
soit souvent implicite. Elle est toutefois exprimée par Arnaud de Villeneuve dans le passage de la Repetitio cité plus haut, puisque le procédé qui ne se conforme pas à la raison est qualifié de "détestable" et de "superstitieux" 24 -un terme fort qui, depuis saint Augustin, va jusqu'à impliquer un pacte tacite avec le démon. Au demeurant, dans l'Epistola de reprobacione nigromantice ficcionis (ou De improbatione maleficiorum) -une œuvre certes à la croisée de la théologie et de la médecine- Arnaud exprime un point de vue démonologique orthodoxe: il réfute notamment la thèse de "certains" qui affirment que l'homme pourrait contraindre les démons "par la vertu de figures qu'ils appellent caractères et par la vertu de sons vocaux qu'ils appellent conjurations"; il affirme qu'il est impossible que ces figures et ses sons aient naturellement des vertus ${ }^{25}$. Au demeurant, le respect assez strict de cette norme de la nondestinativité est l'un des arguments forts que l'on peut opposer à l'attribution tardive à Arnaud du De sigillis, ce texte proposant la fabrication de douze sceaux zodiacaux requérant nombre d'inscriptions, de récitations de psaumes et d'autres incantations ${ }^{26}$.

Certains procédés sans être explicitement destinatifs semblent défier la rationalisation, tel le procédé que l'on trouve dans un traité sur la peste du XVe siècle et qui préconise d'appliquer sur le bubon du pestiféré, tous les septièmes d'heure durant une journée, un coq noir ayant l'anus fendu qui attirera à lui le poison jusqu'à en mourir (soit en tout cent soixante-huit coqs). L'usage d'animaux vivants, tels les escargots, comme cataplasmes appliqués sur les bubons est attesté dans d'autres écrits sur la peste. La propriété occulte peut très bien rendre implicitement compte de tels procédés, mais comment expliquer la couleur des coqs et le chiffre sept, qui sont sans doute les résidus d'une pratique rituelle? ${ }^{27}$

Pourtant même les incantations, pourtant par définition éminemment destinatives, ont pu recevoir des tentatives de justification rationnelle non destinative via la seconde voie possible évoquée plus haut, celle du pouvoir de l'imagination.

\footnotetext{
${ }^{24}$ M. McVaugh, Incantationes in Late Medieval Surgery, p. 336.

25 "Dicunt enim quidam quod virtute figurarum quas vocant karacteres et virtute vocum quas vocant coniurationes vel coniuria compelluntur" (éd. de S. Giralt, dans Arnaldi de Villanova, Opera medica omnia, VII.1, p. 228); sur la délimitation du licite, voir aussi S. Giralt, Estudi introductori, ibidem, pp. 138-141.

${ }^{26}$ N. Weill-Parot, Astrologie, médecine et art talismanique. Le De sigillis a été édité par G. Federici Vescovini, Sigilli cosiddetti arnaldiani.

${ }^{27}$ L'expérience avec les coqs se trouvent dans un Tractatus de peste sananda ad rectores Erfordenses, rédigé en 1405 à Erfurt et édité par K. Sudhoff, Pestschriften aus den ersten 150 Jahren, pp. 85-86, cf. N. Weill-Parot, La rationalité médicale, pp. 84-85. L'expérience avec les escargots figure dans un traité de Raimond Chalmel édité par D. Le Blévec, Un médecin vivarois.
} 


\section{LA RATIONALISATION DES CHARMES ET DES INCANTATIONS PAR L'IMAGINATION ET SES LIMITES}

La solution du pouvoir de l'imagination sur le propre corps de celui qui imagine, qui maintient une forme de contiguïté exigée par la physique aristotélicienne, est-elle une rationalisation aussi satisfaisante eu égard aux intentions de ses utilisateurs que celle recourant aux propriétés occultes? Il ne semble pas, pour au moins trois grandes raisons. La première ne concerne pas le médecin en tant que médecin, mais en tant qu'éventuel partisan de la notion de magie naturelle. Les trois façons de rendre compte de l'action de l'imagination aboutissent toutes à une naturalisation insatisfaisante du phénomène magique du point de vue logique: postuler l'action à distance est enfreindre un axiome de base de la physique aristotélicienne (celui du contact entre moteur et mû); placer dans l'intermédiaire un vecteur comme les species, ces simulacres (originellement définis en optique) en eux-mêmes immatériels mais tirant du milieu qu'elles traversent (l'air) leur matérialitée ${ }^{28}$, est résoudre d'une certaine façon le fossé entre la cause et le causé et donc, par là même, faire disparaître la part d'inexpliqué constitutive de la magie; enfin ramener l'explication à un contact entre l'imagination du patient et son propre corps consiste, là encore, à revenir à une explication qui fait disparaître toute part occulte, et donc tout aspect magique -la magie ne tenant qu'à l'ignorance -toujours fragile- du patient.

Cela conduit à la deuxième raison qui renvoie à l'éthique médicale elle-même, puisque la cause d'une telle thérapie repose, en quelque sorte, sur le pieux mensonge que le médecin, qui connaît les vraies raisons agissantes, fait croire à son patient. Si, d'un côté, Roger Bacon condamne la supercherie qui consisterait à se faire passer pour un magicien aux yeux du peuple qui ignorerait que l'aimant attire le fer, en ajoutant des caractères et des incantations, d'un autre côté il approuve l'emploi de ces mêmes caracteres et carmina, même fictifs, dans la perspective thérapeutique du De physicis ligaturis de Qustā ibn Lūqā, ce texte traduit par Constantin l'Africain, qui présente des amulettes et des incantations dont l'efficacité est en grande partie justifiée par la confiance que le malade place en elles (et même si ailleurs Bacon reconnaît bien une efficacité réelle des paroles ${ }^{29}$. Cette justification repose aux yeux de Bacon sur deux fondements: d'une part l'utilité pratique et de l'autre la conformité de cette dissimulation avec le processus naturel lui-même ${ }^{30}$.

${ }^{28}$ Voir notamment: D.C. Lindberg, Theories of Vision; K. Tachau, Vision and Certitude; L. Spruit, Species intelligibilis from Perception.

${ }^{29}$ Sur le De physicis ligaturis de Qustā ibn Lūqā, voir infra note 43.

${ }^{30} \mathrm{~N}$. Weill-Parot, Encadrement ou dévoilement. 
Arnaud de Villeneuve, en revanche, semble admettre simplement en passant un "discreto engaño del paciente" (pour reprendre l'expression de Juan Paniagua) dans le seul but d'accroître la confiance de celui-ci dans la guérison, mais ne paraît guère priser le recours à ce type de procédé sollicitant l'imagination du patient ${ }^{31}$. Le De parte operativa d'Arnaud de Villeneuve, censé originellement aller de la tête aux pieds mais dont seule la partie consacrée aux maladies de la tête a été rédigée, comprend l'usage de nombreux empirica de saveur magique, mais même pour les maladies mentales -du reste, envisagées d'un point de vue strictement organique ${ }^{32}$ - les procédés mettent avant tout en jeu les propriétés occultes et non le pouvoir de l'imagination sur son propre corps. Par exemple, pour l'aliénation, il faut, entre autres, enlever les causes premières et notamment supprimer les maléfices qui ont été perpétrés si on en a connaissance "en chassant les choses occultes par des choses qui permettent d'y faire face grâce à leur propriété, comme des pierres précieuses ou des parties de plantes ou d'animaux ou des sceaux présentant des figures célestes suspendus ou accrochés au malades ou situés de façon convenable dans les meubles, les couvertures ou la maison" ${ }^{33}$. On reconnaît dans la dernière prescription l'usage des "images astrologiques".

Le troisième motif d'insatisfaction pour cette solution par le pouvoir de l'imagination sur le corps de celui qui imagine est de nature théologique, puisqu'il met en jeu les implications du critère de la destinativité. Certes le médecin n'est pas un théologien et la médecine a manifesté une remarquable autonomie intellectuelle à l'égard de la théologie. Mais, on l'a dit, cette autonomie ne signifiait pas une indépendance susceptible de heurter de front les interdits théologiques les plus profonds. Dans un passage, relevé par Danielle Jacquart, de l'avis rendu par les médecins de la faculté de médecine de Paris en 1348 sur la peste, les médecins font coïncider la foi et la science en renforçant par une interprétation physiologique le fait de "faire la paix avec Dieu". Il s'agit en effet d'éviter qu'à cause de la peur l'esprit vital contaminé par l'air empoisonné ne soit attiré vers le cœur et de provoquer, au contraire, un "mouvement centrifuge" de cet esprit, ce qui peut être réalisé par la joie ou par le fait de ne plus craindre la mort ${ }^{34}$. Certes, dans ce cas, il s'agit pour les

\footnotetext{
${ }^{31}$ J.A. Paniagua, La psicoterapia en las obras médicas, pp. 435-436 et note 35.

${ }^{32}$ Sur l'approche médicale de la folie au Moyen Âge, voir notamment: D. Jacquart, La réflexion médicale; eadem, Avicenne et la nosologie galénique.

33 "occulta vero reprimendo rebus obviantibus a proprietate, ut mineralibus aut partibus plantarum aut animalium vel sigilis [sic!] celestium figurarum suspensis egro vel colligatis vel competenter situatis instrumentis vel operimentis aut mansione" (Arnaldi de Vilanova, De parte operativa, dans idem, Opera, f. 128v).

${ }^{34}$ D. Jacquart, La médecine médiévale, p. 238 et note 25 , cf. Avis édité par É. Rebouis, Étude historique et critique, p. 114.
} 
médecins, comme l'écrit D. Jacquart, “de rappeler leur piété, tout en livrant un message rationalisant". Néanmoins, l'horizon d'une naturalisation de la prière, par exemple, ne serait pas sans danger théologique. En d'autres termes, proposer une lecture naturaliste des incantations via la doctrine -parfaitement acceptable par ailleurs- du pouvoir de l'imagination sur son propre corps pouvait brouiller le critère, fondamental, de la destinativité en s'en prenant au cœur même de la relation entre le fidèle et Dieu. Ce n'est sans doute pas un hasard si Roger Bacon qui a autant de facilité à approuver l'usage des incantations fondées sur la croyance du malade, est aussi un auteur qui, contrairement à l'orthodoxie dominante qui condamne toute pratique destinative, propose de "naturaliser" le pouvoir des mots en se fondant sur une forme de rayonnement chez eux qui rappelle la doctrine néoplatonicienne du De radiis d' al-Kindīis ${ }^{35}$.

Que la rationalisation par le pouvoir interne au corps de l'imagination fût insatisfaisante pour un médecin tenant de la magie naturelle et qu'elle entrât en conflit avec la rationalisation par les propriétés occultes et l'astrologie est montré par un passage intéressant de l'œuvre d'un médecin de la fin du $\mathrm{XV}^{\mathrm{e}}$ siècle, resté fidèle à la scolastique médicale médiévale.

\section{LES HÉSITATIONS D’UN MÉDECIN FACE À UN EMPIRICUM DIFFICILE}

Jeroni Torrella (1456-après 1502) est d'origine valencienne, mais s'est formé en Italie où il eut pour maître notamment Pierleone de Spolète. Lecteur du De vita de Marsile Ficin qu'il cite sans le nommer dans son Opus praeclarum de imaginibus astrologicis $(1496-1500)^{36}$, il n'en reste pas moins scrupuleusement fidèle au cadre péripatéticien et galénique de la scolastique médiévale et peut, à ce titre, illustrer le degré d'approfondissement atteint dans la réflexion sur les empirica dans ce cadre hérité et respecté qui, de son temps, est concurrencé par de nouveaux courants -notamment néoplatoniciens. Son Opus praeclarum, qui était destiné à analyser la validité et la licéité de l'usage des "images astrologiques" à des fins thérapeutiques, se réfère essentiellement à la doctrine de la forme spécifique et des propriétés occultes dans une gradation qui va de simples corps naturels utilisés comme amulette, telle la pierre d'aigle contre les fausses couches qu'il dit avoir prescrite avec succès à la comtesse de Cocentaina, en passant par les médicaments astrologiques, mélanges d'ingrédients effectués sous une configuration astrologique adéquate, jusqu' aux "images astrologiques", en particulier le sceau

${ }^{35}$ Sur le pouvoir des mots chez Bacon, voir I. Rosier, La Parole comme acte; sur les réflexions médiévales autour du pouvoir des mots: B. Delaurenti, La Puissance des mots.

${ }^{36}$ V. Perrone Compagni, Le immagini del medico Gerolamo Torrella. 
du Lion imprimé dans de l'or quand le soleil est dans le Lion et utile contre le calcul rénal. La doctrine astrologique des propriétés occultes accidentelles sur laquelle repose toute la démonstration "albertiste" favorable aux " images astrologiques " telle que la présente Torrella -et à laquelle il souscrit très visiblement, même s'il n'ose l'affirmer par prudence théologique- est contradictoire avec celle qui se fonderait sur le pouvoir de l'imagination sur le corps de celui qui imagine. C'est ce que dit expressément Torrella lui-même à la fin de son traité après avoir rappelé cette doctrine en s'appuyant sur les Problemata aristotéliciens et sur Avicenne, en citant l'exemple devenu classique de la vue d'un homme qui bâille provoquant un bâillement ou celui des ergots qui poussent chez une poule qui a vaincu un $\operatorname{coq}^{37}$. Il affronte cette doctrine explicative concurrente:

Quelqu'un peut-être pourrait dire: si la confiance du malade ou une pensée favorable de sa part à l'égard du remède élaboré en une constellation adéquate est la cause de la santé, la seule pensée ou confiance pourra donc être cause de la santé sans le remède; on peut ainsi conclure que cette introduction de la santé ne doit pas être réduite à la qualité introduite par les corps supérieurs en une constellation déterminée ${ }^{38}$.

La causalité par le pouvoir interne au corps de l'imagination rend inutile le procédé astrologique, qu'il s'agisse d'un médicament astrologique ou d'une "image astrologique". Torrella répond en s'abritant derrière cet Albert le Grand qui est, à ses yeux, le représentant des partisans des "images astrologiques" opposé à Thomas d'Aquin qui en est l'adversaire - une distinction, du reste, qui est historiquement juste:

À cela Albert répondrait que la confiance du malade au sujet de cette qualité céleste ne nuit pas, et même aide, à l'introduction de la santé et peut-être même l'accélère; mais que la confiance soit la cause totale et principale de l'introduction de la santé cela ne doit pas être admis. Il faut au contraire soutenir que la qualité causée en une certaine constellation des orbes est le principe quo et plus fondamental pour l'introduction de la santé que la relation à la confiance éprouvée < par le patient $>$. Mais il ne faut nullement nier que la confiance aide un petit peu à cette introduction de la bonne santé du corps humain.

\footnotetext{
${ }^{37}$ Sur des exemples classiques d'action à distance: B. Delaurenti, La fascination et l'action.

38 "Dicet forte aliquis: Si confidentia egrotantis et cogitatio bona eius de medicina in constellatione debita facta est causa sanitatis, ergo sola cogitatio aut confidentia absque medicina poterit esse sanitatis causa; quare concludi potest hanc sanitatis inductionem nequaquam esse reducendam in qualitatem a corporibus superioribus in certa constellatione inductam" (J. Torrella, Opus praeclarum, concl., p. 242).
} 
C'est pourquoi ceux qui suivent cette opinion jugeraient qu'il faut retirer la première conséquence et par conséquent la seconde $^{39}$.

Dans la quatrième partie de son traité où il répond (au nom des disciples d'Albert) aux arguments hostiles aux "images astrologiques" énoncés dans la seconde partie (au nom des "Thomistes"), Torrella cite des exemples de choses qui acquièrent (ou perdent) des vertus sans être "substantiellement transmuées" mais seulement "accidentellement": ainsi, le diamant approché d'un aimant lui retire sa vertu attractive du fer. Il est alors confronté à un empiricum dont il a bien du mal à rendre compte:

En outre, les fils surtout s'ils sont teints avec du murex marin qui ont étranglé une vipère apportent contre l'angine une aide digne d'admiration et qui surmonte la dangerosité de ce qui est craint, écrivent Galien et Avicenne, qui furent très savants et eurent l'expérience de nombreuses choses ${ }^{40}$.

Torrella oppose ensuite deux interprétations du phénomène:

Un praticien (artista) prend un fil et étrangle avec lui une vipère. Mais que la nature toute seule ne peut effectuer cela est suffisamment assuré. Or une vertu de ce genre ne peut être réduite à des causes manifestes. Et en effet, nous échappe de quelle manière cela en particulier advient, c'est pourquoi il faut la réduire à la toute propriété (totam proprietatem). Mais les tenants de l'opinion contraire diront que ces choses sont superstitieuses et appartiennent au démon puisqu'on ignore les causes qui produisent un tel effet que seul le Christ connait et révèle à qui il veut, ou ils diront que cet effet doit être réduit à la confiance du malade qui souffre d'angine. À ceux-là ceux qui suivent l'opinion d'Albert répondront qu'un effet de ce type ne peut nullement être réduit à une astuce du démon, mais seulement à une propriété occulte ${ }^{41}$.

\footnotetext{
39 "Cui diceret Albertus confidentiam egrotantis de tali qualitate caelesti non nocere, imo iuuare ad sanitatem et forte celeriter inducendam; sed quod confidentia sit totalis causa et principalior ad sanitatem inducendam non est admittendum. Imo potius est tenendum quod qualitas in certa orbium constellatio ecausata est principium quo et principalius ad sanitatem inducendam, relatione ad confidentiam habita. Confidentiam autem aliquantulum iuuare ad talem corporis humani bonam valitudinem inducendam, minime est negandum. Quamobrem primam consequentiam, et consequenter secundam, interimendam esse iudicarent hanc opinionem tenentes" (ibidem, pp. 242-243).

40 "Praetera fila praesertim murice marino tincta quae viperam suffocarunt et iuuamentum admiratione dignum pertransiensque magnitudinem eius quod timetur in squinantia conferre scribunt Galienus et Avicenna doctissimi et multarum rerum experientiam habentes" (ibidem, IV, ad 20, p. 203).

41 "Artista sumit philum et eo viperam suffocat. Sed satis compertum est naturam se sola hoc non facere. Non autem generis huius virtus reduci potest in causas manifestas. Nos etenim
} 
Dans ce passage, Torrella explicite bien la fonction d'explication ultime dévolue à la tota proprietas (ou propriété occulte). De plus, il attribue aux adversaires du talisman naturaliste reposant sur l'usage astrologique des propriétés occultes une double thèse: soit l'explication démonologique, soit celle qui a recours au pouvoir de l'imagination sur son propre corps -ce qui est une manière de considérer que cette seconde voie ne permet pas de fonder une magie naturelle à laquelle les "thomistes" sont hostiles. Torrella suit de de très près la formulation du Canon, où Avicenne range clairement cette expérience au nombre des choses éprouvées qui agissent par leur propriété (ex rebus expertis que faciunt sua proprietate) ${ }^{42}$.

Le fait que cet exemple appelle aussi chez Torrella une référence à la doctrine de la confiance du malade tient peut-être ici à une autre source: le $D e$ physicis ligaturis de Qustā ibn Lūqā, où est aussi mentionné Galien (avec un titre d'ouvrage qui est inconnu) et où on lit en effet:

Or dans le livre du même [i.e. Galien] la Médecine des médecins, il dit: "Si le cou d'un serpent de Tyr est étranglé avec un fil de lin, et que tu suspends <ce fil > au cou de quelqu'un qui suffoque, il est libéré de sa suffocation et cela est très efficace contre les abcès à la gorge" ${ }^{\prime 43}$.

Fidèle cependant à la doctrine selon laquelle des choses peuvent acquérir des vertus à la suite d'une mutation accidentelle et non substantielle, Torrella tente une explication du pouvoir de ce fil:

fugit quo pacto istud particulariter fiat quamobrem in totam proprietatem reducere oportet. Dicent autem adversam tenentes sententiam haec superstitiosa et ad daemonem pertinentia esse postquam causas ignorant talis effectus productiuas quas solus Christus scit et quibus vult reuelat, aut dicent in confidentiam patientis squinantiam talem effectum esse reducendum. Quibus dicent Alberti sententiam sequentes huiusmodi effectum nequaquam in demonis astuciam reducendum esse, sed in occultam dumtaxat proprietatem" (ibidem, pp. 203-204).

42 "iuvamentum maximum est ut sumantur fila ex proprie tincta cum murice marino et prefocetur cum eis vipera deinde circundetur eis collum eius qui habet aliquid horum apostematum. Illud enim confert ei iuvamentum mirabile bonum pertransiens quantitatem eius quod timetur" (Avicenna, Canon, III. 9. 1. 10, f. L 6r col. 1-2).

43 "In libro autem eiusdem medicorum medicina ait: «Quia si serpentis tyriae suffocaturi collum cum filo lineo, suffocandi collo suspenderis, absolvitur a suffocatione sui et multum valet in apostematibus faucium»" (R. Casazza (éd.), El De physicis ligaturis, p. 107). J'ai changé un peu la ponctuation, parce que "cum filo lineo" me semble aller avec "suffocaturi" et que c'est le fil qui est ensuite suspendu. De ce point de vue je m'écarte aussi de la traduction proposée par J. Wilcox et J.M. Riddle (Qustā ben Lūqua 's, p. 44): "Also in the book by the same called Medicine of Physicians he says that if after strangling a Tyrian serpent, you suspend its neck with a linen thread about the neck of someone who is about to suffocate, he is relieved from suffocating and it is [also] very efficacious for abscesses of the throat". Les éditeurs précisent que cet ouvrage de Galien n'a pu être identifié. 
Le fil, non mué substantiellement, appliqué au cou de la vipère, d'une manière qui nous est cachée (occulto), obtient une force occulte (occultam) mais prouvée par son effet ${ }^{44}$.

Cette explication privilégie in fine les propriétés occultes, connaissables seulement par l'expérience de leur effet, qui demeurent la principale explication rationnelle des empirica dans la scolastique médicale, qu'ils soient magiques ou non.

En ce sens, Torrella, à la fin du XVe siècle, parcourt les voies possibles de la rationalisation naturaliste des empirica propre à la scolastique médicale et tranche en faveur de la solution dominante: celle qui a recours à la forme spécifique ou tota substantia et aux propriétés occultes qui en découlent. Il témoigne ainsi, à une date tardive, de la fonction à la fois discriminante et intégratrice du raisonnement scientifique scolastique qui, d'un côté, délimite clairement le champ naturaliste (en rejetant toute destinativité) et, de l'autre, tente de pousser le plus loin possible la causalité naturelle à l'intérieur du cadre péripatéticien et galénique. Ce rationalisme scientifique particulièrement cohérent entre 1250 et 1320 demeure à l'état de tendance dans la pensée scolastique bien plus longuement et reste intacte chez certains représentants tardifs de ce savoir universitaire.

\section{BIBLIOGRAPHIE CITÉE}

Agrimi, Jole; Crisciani, Chiara, Medici e "vetulae" dal Duecento al Quattrocento: Problemi di una ricerca, dans Cultura popolare e cultura dotta nel Seicento, Milan, F. Angeli, 1983, pp. 144-159.

Arnaldus de Villanova, Opera, Lyon, S. de Gabiaono, 1532.

Avicenna, Canon, Venise, per Simonem Papiensem, 1500.

Boghossian, Paul, La peur du savoir: sur le relativisme et le constructivisme de la connaissance, traduit de l'anglais par Deroy, Ophelia; préface et annexes de Rosat, Jean-Jacques, Marseille, Agone, 2009.

Boudon, Raymond, Croire et savoir: penser le politique, le moral et le religieux, Paris, PuF, 2012.

Boudon, Raymond, La rationalité, Paris, Presses universitaires de France, 2012.

\footnotetext{
44 "Filum, vt ad exemplum superius deductum redeamus, substantialiter non mutatum, collo vipere applicatum, modo nobis occulto vim occultam sed effectu comprobatam nanciscitur" (J. Torrella, Opus praeclarum, p. 205).
} 
Casazza, Roberto (éd.), El De physicis ligaturis de Costa ben Luca: Un tratado poco conocido sobre el uso de encantamientos y amuletos con fines terapéuticos, "Patristica et Mediaevalia" 27 (2006), pp. 87-113.

Chandelier, Joël, Expérience, expérimentation et connaissance dans la médecine scolastique italienne du 14e siècle, dans Bénatouil, Thomas; Draelants, Isabelle (éd.), Expertus sum. L'expérience par les sens dans la philosophie naturelle médiévale, Florence, SISMEL Edizioni del Galluzzo, 2011, pp. 385-403.

Copenhaver, Brian P., Scholastic Philosophy and Renaissance Magic in the De vita of Marsilio Ficino, "Renaissance Quarterly" 37 (1984), pp. 523-554.

Daston, Lorraine; Park, Katharine, Wonders and the Order of Nature (11501750), New York, Zone Books, 1998.

Delaurenti, Béatrice, La fascination et l'action à distance: questions médiévales (1230-1370), "Médiévales" 50 (2006), pp. 137-154.

Delaurenti, Béatrice, La puissance des mots. "Virtus verborum". Débats doctrinaux sur le pouvoir des incantations au Moyen Âge, Paris, Cerf, 2007.

Eamon, William; Keil, Gundolf, "Plebs amat empirica": Nicholas of Poland and His Critique of the Medieval Medical Establishment, "Sudhoffs Archiv" 17 (1987), pp. 180-196.

Federici Vescovini, Graziella, Sigilli cosiddetti arnaldiani, "Traditio" 60 (2005), pp. 201-242.

Federici Vescovini, Graziella, La concezione della virtus occulta nella dottrina medica di Arnaldo di Villanova e di Pietro d'Abano, dans Hamesse, Jacqueline; Weijers, Olga (éd.), Écriture et réécriture des textes philosophiques médiévaux. Volume d'hommage offert à Colette Sirat, Turnhout, Brepols, 2006, pp. 107-135.

Gansyniec, Ryszard, Brata Mikołaja z Polski pisma lekarskie, Poznań-Cracovie, Gebethner, 1920.

Giralt, Sebastià (ed.), Epistola de reprobacione nigromantice ficcionis (de improbatione maleficiorum), Barcelona, Universitat de Barcelona Fundació Noguera, 2005. (Arnaldi de Vilanova Opera Medica Omnia; VII.1).

Giralt, Sebastià, Proprietats: Las propiedades ocultas según Arnau de Vilanova, "Traditio" 63 (2008), pp. 327-360.

Guido de Caulhiaco, Chirurgia Chirurgia magna, McVaugh, Michael (éd.), Leyde, E. J. Brill, 1997.

Hutchison, Keith, Dormitive Virtues, Scholastic Qualities, and the New Philosophies, "History of Science" 29 (1991), pp. 245-278. 
Jacquart, Danielle, La réflexion médicale médiévale et l'apport arabe, dans Postel, Jacques; Quétel, Claude (éd.), Nouvelle histoire de la psychiatrie, Toulouse, Privat, 1983, pp. 43-53; nouv. éd., Paris, Dunod, 1994, pp. 37-47.

Jacquart, Danielle, La scolastique médicale, dans Grmek, Mirko (éd.), Histoire de la pensée médicale en Occident. 1. Antiquité et Moyen Âge, Paris, Seuil, 1995, pp. XXX.

Jacquart, Danielle, Avicenne et la nosologie galénique: l'exemple des maladies du cerveau, dans Hasnawi, Ahmad; Elamrani-Jamal, Abdelali; Aouad, Maroun (éd.), Perspectives arabes et médiévales de la tradition scientifique et philosophique grecque, Louvain, Peeters - Paris, Institut du monde arabe 1997, pp. 217-226.

Jacquart, Danielle, La médecine médiévale dans le cadre parisien, XIVe-XVe siècle, Paris, Fayard, 1998.

Johnsson, J.W.S., Les “Experimenta Magistri Nicolai” publiés pour la première fois, "Bulletin de la Société française d'histoire de la médecine" 10/6 (1911), pp. 269-290.

Keil, Gundolf, Virtus occulta: Der Begriff des "empiricum” bei Nikolaus von Polen, dans Buck, August (éd.), Die okkulte Wissenschaften in der Renaissance, Wiesbaden, Komm. Otto Harrassowitz, 1992, pp. 159-196.

Kieckhefer, Richard, The Specific Rationality of Medieval Magic, "American Historical Review" 99 (1994), pp. 813-836.

Le Blévec, Daniel, Un médecin vivarois à la cour pontificale d'Avignon: Raimond Chalmel, "Revue du Vivarais" 90 (1986), pp. 13-24.

Lindberg, David C., Theories of Vision from al-Kindi to Kepler, Chicago, The University of Chicago Press, 1976.

Mauss, Marcel; Hubert, Henri, Esquisse d'une théorie générale de la magie, "L'Année sociologique" (1902-1903), rééd. dans Mauss, Marcel, Sociologie et Anthropologie, Paris, PuF, 1950, rééd. 1997, pp. 1-141.

McVaugh, Michael, Incantationes in Late Medieval Surgery, dans Marchetti, Giancarlo; Rignani, Orsola; Sorge, Valeria (éd.), Ratio et Superstitio: Essays in Honor of Graziella Federici Vescovini, Louvain-la-Neuve, Federation Internationale des Instituts d'Études médiévales, 2003, pp. 319-345.

McVaugh, Michael, The Rational Surgery of the Middle Ages, Florence, SISMEL Edizioni del Galluzzo, 2006.

Paniagua, Juan A., La psicoterapia en las obras médicas de Arnau de Vilanova, dans Paniagua, Juan A., Studia Arnaldiana. Trabajos en torno a la obra médica de Arnau de Vilanova, c. 1240-1311, Barcelone, Fundación Uriach 1838, 1994, pp. 425-447. 
Perrone Compagni, Vittoria, Le immagini del medico Gerolamo Torrella, “Annali dell'Istituto di filosofia dell'Università di Firenze, Facoltà di Lettere e Filosofia", 1 (1979), pp. 17-45.

Perrone Compagni, Vittoria, "Artificiose operari". L'immaginazione di Avicenna nel dibattito medievale sulla magia, dans Bettetini, Maria; Paparella, Francesco (éd.); Furlan, Roberto (collab. éd.), Immaginario e immaginazione nel Medioevo, Louvain-la-Neuve, Federation Internationale des Instituts d'Études médiévales, 2009, pp. 271-296.

Rebouis, Émile, Étude historique et critique sur la peste, Paris, Picard, 1888.

Rosier, Irène, La parole comme acte. Sur la grammaire et la sémantique au XIIIe siècle, Paris, Vrin, 1994.

Sanchez, Pascal, La rationalité des croyances magiques, Genève, Droz, 2007.

Spruit, Leen, Species intelligibilis from Perception to Knowledge, vol. I: Classical Roots and Medieval Discussions, Leyde, E. J. Brill, 1994.

Sudhoff, Karl, Pestschriften aus den ersten 150 Jahren nach der Epidemie des "Schwartzen Todes", "Archiv für Geschichte der Medizin" 11/3-4 (1919), pp. 75-92 (núm. 114-115).

Tachau, Katherine, Vision and Certitude in the Age of Ockham. Optics, Epistemology and the Foundation of Semantics 1250-1345, Leyde, E. J. Brill, 1988.

Torrella, Jérôme, Opus praeclarum de imaginibus astrologicis, Weill-Parot, Nicolas (éd.), Florence, SISMEL Edizioni del Galluzzo, 2008.

Weill-Parot, Nicolas, Les "images astrologiques" au Moyen Âge et à la Renaissance. Spéculations intellectuelles et pratiques magiques (XIIeXVe siècle), Paris, H. Champion, 2002.

Weill-Parot, Nicolas, Astrologie, médecine et art talismanique à Montpellier: les sceaux astrologiques pseudo-arnaldiens, dans Le Blévec, Daniel (éd.); Granier, Thomas (collab.), L'université de médecine de Montpellier et son rayonnement (XIIIe-XVe siècles), Turnhout, Brepols, 2004, pp. 157-174.

Weill-Parot, Nicolas, La rationalité médicale à l'épreuve de la peste: médecine, astrologie et magie (1348-1500), "Médiévales" 46 (2004), pp. 73-88.

Weill-Parot, Nicolas, Science et magie au Moyen Âge, dans Hamesse, Jacqueline (éd.), Bilan et Perspectives des études médiévales (1993-1998). Actes du IIe Congrès Européen d'Etudes Médiévales, Turnhout, Brepols, 2004, pp. 527-559.

Weill-Parot, Nicolas, Encadrement ou dévoilement: l'occulte et le secret dans la nature chez Albert le Grand et Roger Bacon, "Micrologus" 14 (2006), pp. 151-170.

Weill-Parot, Nicolas, Astrology, Astral Influences and Occult Properties in the Thirteenth and Fourteenth Centuries, "Traditio" 65 (2010), pp. 201-230. 
Weill-Parot, Nicolas, Réalité ou vacuité du mal ? Vaines superstitions, magie et démons au crible de la théologie et de la philosophie naturelle (XIIIe-XIVe siècle), dans Rigaux, Dominique; Russo, Daniel; Vincent, Catherine (éd.), Expériences religieuses et chemins de perfection dans l'Occident médiéval. Études offertes à André Vauchez par ser élèves, Paris, Académie des inscriptions et belles lettres, 2012, pp. 217-232.

Wilcox, Judith; Riddle, John M., Qustā ben Lūqā's Physical Ligatures and the Recognition of the Placebo effet, "Medieval Encounter" 1 (1995), pp. $1-50$.

Fecha de recepción del artículo : noviembre 2012

Fecha de aceptación y versión final : mayo 2013 